|| ISSN(online): 2589-8698 || ISSN(print): 2589-868X || International Journal of Medical and Biomedical Studies

Available Online at www.ijmbs.info

PubMed (National Library of Medicine ID: 101738825) Index Copernicus Value 2018: 75.71

Original Research Article

Volume 3, Issue 5; May: 2019; Page No. 137-141

\title{
SERUM ALBUMIN LEVEL IN PERSISTENT ORGAN FAILURE IN ACUTE PANCREATITIS
}

\author{
${ }^{1}$ Dr. Shefali Mehta, ${ }^{2} \mathrm{Dr}$. Shuchi Goyal, ${ }^{3} \mathrm{Dr}$. Rajendra Triloki, ${ }^{4} \mathrm{Dr}$.A.K. Verma \\ ${ }^{1}$ Assistant Professor, ${ }^{2}$ Professor and Head, ${ }^{3}$ Final year Resident, ${ }^{4}$ Senior Professor, \\ Department of Biochemistry, Rabindranath Tagore Medical College, Udaipur, Rajasthan
}

Article Info: Received 26 April 2019; Accepted 20 May. 2019

DOI: https://doi.org/10.32553/ijmbs.v3i5.232

Corresponding Author: Dr. Shuchi Goyal, Professor and Head, Department of Biochemistry, Rabindranath Tagore Medical College, Udaipur, Rajasthan

Conflict of interest: No conflict of interest.

\section{Abstract:}

Introduction: The research emphasises on the association bond of Serum Albumin Level used as a marker for diagnosis of Persistent Organ Failure in Acute Pancreatitis.

Material and Methods: The samples for the study were collected from the central lab, out-patient department and in-patient department of Department of Biochemistry, adult patients suffering from Acute Pancreatitis correlated to tropical medicinal stream and Gastroenterology Rabindranath Tagore Medical College, Udaipur, Rajasthan The research was carried out on a total of 100 patients for 1 year which were satisfying Atlanta criteria for Acute Pancreatitis will be diagnosed based on the following symptoms; elevated serum amylase, and lipase levels, that is greater than the normal limit, findings associated with the characteristics of the radiological findings. Also, with the abdominal computerized ultrasonography and tomography

Results: In the present research, albumin always descends obviously in AP patients with POF $(p<0.05)$. The AUC under ROC line is 0.869 . Albumin has been proved as an excellent marker of POF in AP. However, no previous study has researched into the association between albumin and incidence of POF in AP. Therefore, this study is the first time to show that the reduction of serum albumin is significantly associated with increased risk of POF in AP.

Conclusion: Thus it can be concluded that serum albumin on admission is independently associated with POF in AP. The study suggests that albumin is a valuable tool for a rapid assessment of POF in patients with AP.

Keywords: Serum Albumin, Persistent Organ Failure, Acute Pancreatitis

\section{Introduction}

Acute pancreatitis is defined as an acute inflammatory process of the pancreas with variable involvement of other regional tissues or remote organ systems. Among the patients with acute pancreatitis (AP) undergoing a general course clinically, $10 \%$ to $20 \%$ of the patients are found with development of persistent failure of organ that lasts for more than 48 hours. AP is associated with the significant mortality rate of around $30 \%$. In depth consideration is recognised with the organ failure patients persistently, and is termed as the key determinant of the severity with presence or absence of local pancreatic complications.
Albumin excessively tends to synthesise in the liver. The low level of serum albumin is often experienced in the elderly patients. Patients with liver cirrhosis, diabetes, heart failure, malnutrition, cancer and also sepsis, coronary heart diseases, acute phase protein, initial serum albumin were also likely to be associated with the liver disease progression. Logistic regression on multivariate basis and the analysis of the subgroup were in totality determined in cases where decreased albumin was found to be associated with mortality rate that tends to take place as a side-effect of organ failure. The low serum albumin was independently associated with the increased risks of development of the persistent organ failure and is said to be the major cause of death in acute pancreatitis. 
Hypoalbuminemia is also termed as a predictor of the acute pancreatitis severity marker.

As the levels of serum albumin decreases, the risk associated with the persistent organ failure increases. The incidence of the organ failure was found to be $3.5 \%, 10 \%$, and $41.6 \%$ successively. It was found in patients with normal to mild albumin and severe hypoalbuminaemia. Severe acute pancreatitis has a high percentage with the incidence of the mortality ratio; whereas the Non-infectious inflammation is caused by the systemic inflammation response syndrome as a result of early onset of inflammation. Most of the patients suffer from mild, self limiting and inflammatory processes. It is vital to determine therapeutic strategies to provide an early assessment of the diseases severity through effective treatment. Invasive and the non-invasive techniques, the scoring system, radiological imaging, bio-chemical parameters are used for diagnosis and evaluation of disease severity in acute pancreatitis ${ }^{3}$.

It is significant to determine the patients suffering from acute pancreatitis at an early stage. Also to makes the patients secured from developing persistent organ failure in the early course of time and helping out with disease recovery. The accuracy of the systems helps in predicting the value associated with persistent organ failure. Complications are highly associated with the optimal management of current resources ${ }^{6}$.

\section{Materials and Methods}

\section{Study Area}

This was a cross sectional observational study comprising of adult patients suffering from Acute Pancreatitis correlated to tropical medicinal stream and Gastroenterology with internal medical departments, at - Department of Biochemistry, Rabindranath Tagore Medical College, Udaipur, Rajasthan in the year 2018-2019. Acute Pancreatitis will be diagnosed based on the following symptoms; elevated serum amylase, and lipase levels, that is greater than the normal limit, findings associated with the characteristics of the radiological findings. Also, with the abdominal computerized ultrasonography and tomography. It is additionally termed and diagnosed as per the classification done on the basis acute pancreatitis, with local systemic complications. Severe acute pancreatitis associated with organ failure, local systemic complications and resolved between 48 hours.
The etiology of AP is associated with the biliary imaging, ultrasonography or computed tomography associated with endoscopic retrograde cholangiopancreatography that deals with gallbladder and stones with the common bile duct. Alcohol induced diagnosis with intake of alcohol to the onset of the symptom that can be established. It is also associated with the Traits of ERCP, hyperlipedemia, drugs, trauma and associated terms. Allied conditions like Idiopathic cases and patients with the evidence of chronic pancreatitis, malignancy, kidney and liver failure, anemia with severe infections present in the last tenure, or terms like organ transplantation were not included.

At the time of the admission and hospitalization, a quick medical history and physical examination was done, like the etiology of the pancreatitis, organ failure history, demographic data, severity associated with the AP index, all were noted and marked carefully. Blood sample and urine sample were collected and included the complete blood count. Tests like WBC, RBC, platelets count, Red cell distribution width, haemoglobin serum albumin levels, serum creatinine level, blood urea nitrogen, serum calcium, sodium, serum potassium, serum glucose etc. (CRP) arterial blood gas analysis and lactate dehydrogenase that is (LDH), etc. were also checked and analysed before proceeding for further treatment.

\section{Study Participants}

\section{Inclusion Criteria}

1) Abdominal pain consistent with $A P$

2) Contrast-enhanced computed tomography (CECT), magnetic resonance imaging (MRI) or abdominal ultrasonography findings characteristic of AP

3) Serum amylase and/or lipase elevation $\geq$ three times the upper limit of normal

\section{Exclusion Criteria}

1) Patients younger than 18-year-old

2) The time from abdominal pain onset to hospital admission $\geq 72 \mathrm{~h}$

3) Chronic pancreatitis

4) Pancreatitis induced by trauma or pregnancy

5) Unavailable laboratory measurements or medical records.

Thus, a total of 100 patients diagnosed with AP were selected for the study. 


\section{Statistical Analysis}

All the significant factors associated with median and inter-quartile range were compared through Mann Whitney $U$ tests Chi-square $\left(\chi^{2}\right)$ and Fisher's exact probability test were performed for making a comparison. The positive-negative predictive test value was determined with the ratio (+LR -LR). The Kaplan Meier method was used to estimate the survival rate and these tests dealt with statistical significance assessed at less than 0.05 value. The factors included with independent predict AP related in-hospital mortality, multiple regression analysis, significant variables and the univariate analysis with $(P<0.05)$.

\section{Observations and Results}

Table 1: Type of POF and corresponding Mortality

\begin{tabular}{|l|l|l|}
\hline & Persistent organ failure & In-hospital mortality \\
\hline Solitary POF & 10 & 9 \\
\hline Respiratory & 10 & 9 \\
\hline Renal & 0 & 0 \\
\hline Cardiovascular & 0 & 0 \\
\hline Multiple POF & 5 & 4 \\
\hline Respiratory + renal & 2 & 2 \\
\hline Respiratory + cardiovascular & 2 & 1 \\
\hline Respiratory + cardiovascular + renal & 1 & 1 \\
\hline Total & 15 & 13 \\
\hline
\end{tabular}

As per above table, 10 patients developed solitary POF (all of respiratory system). On the other hand, 5 patients developing multiple POF ( 2 of lung and kidney, 2 of lung and heart, and 1 of lung, kidney and heart). 13 patients with POF died during hospitalization. No death was observed in patients without POF.

Table 2: Comparison between patients with and without POF

\begin{tabular}{|l|l|l|l|}
\hline & Without POF, $\mathrm{n}=35$ & With POF, $\mathrm{n}=15$ & $\mathrm{P}$-Value \\
\hline Sex (M/F) & $26-\mathrm{M}, 9-\mathrm{F}$ & $11-\mathrm{M}, 4-\mathrm{F}$ & NS \\
\hline Age (Year) & 48.5 & 49.2 & NS \\
\hline ALB (g/L) & 37.9 & 29.1 & 0.000 \\
\hline ALT (U/L) & 38.2 & 39.9 & NS \\
\hline AST (U/L) & 35.9 & 44.8 & NS \\
\hline LDH (U/L) & 249 & 468 & 0.000 \\
\hline ALP (U/L) & 75.9 & 72.3 & NS \\
\hline
\end{tabular}

Compared to patients without POF, patients with POF showed significantly elevated values of LDH, whereas the levels of ALB were statistically lower

Table 3:

\begin{tabular}{|l|l|l|l|l|}
\hline & $\begin{array}{l}\text { Univariate Analysis } \\
\text { Odd Ratio }\end{array}$ & P-Value & $\begin{array}{l}\text { Multivariate Analysis } \\
\text { Odd Ratio }\end{array}$ & P-Value \\
\hline Age (Year) & 1.009 & NS & - & - \\
\hline ALB (g/L) & 0.729 & 0.000 & $0.751(0.644,0.865)$ & 0.000 \\
\hline LDH (U/L) & 1.01 & 0.000 & - & - \\
\hline
\end{tabular}

In univariate analysis, ALB and LDH were found to be significantly associated with the incidence of POF. According to Multivariate Analysis, ALB remained an independent prognostic factor for POF in AP (OR: 0.751, $95 \% \mathrm{Cl}: 0.644-0.865 ; p<0.05)$ 
Table 4:

\begin{tabular}{|l|l|l|l|l|l|l|}
\hline & AUC $(95 \% \mathrm{Cl})$ & Cut-off & Sensitivity & Specificity & PPV & NPV \\
\hline ALB & 0.869 & 32.9 & 0.785 & 0.829 & 0.651 & 0.9 \\
\hline
\end{tabular}

ALB on admission was shown to have an area under curve (AUC) of the receiver operating characteristic of 0.869 (95\% Cl: $0.805-0.935)$, with specificity of $82.9 \%$, a sensitivity of $82.9 \%$, positive predictive value (PPV) of $65.1 \%$, and negative Predictive value (NPV) of $90.0 \%$. The optimal threshold was $32.9 \mathrm{~g} / \mathrm{L}$.

\section{Discussion}

Acute pancreatitis is one of the most serious emergencies in abdominal surgery department. The proposed design is based on 2012 revision of the Atlanta Classification of acute pancreatitis, and found that the level of albumin in acute pancreatitis patients with POF is much lower than patients without POF.

Albumin is a stable and very flexible heart-shapemolecule. It's a natural plasma protein with median half-life of 18 to 19 days and synthesized only by liver at a rate of 9 to $14 \mathrm{~g}$ per day in healthy individuals.

Albumin has well-known effects on maintaining fluid balance, being responsible for 75 to $80 \%$ of colloid osmotic pressure in the basal physiological state. The binding characteristic of Albumin to an extremely wide range of endogenous and exogenous ligands, enables it to transport them to specific tissues and organs, to increase their solubility in plasma, or to dispose of them when they are toxic.

In the progress of many diseases, infection, inflammation, a low-level of serum albumin has been identified. It is because of abnormal metabolism of albumin led by inflammation response, as well as a low intake of albumin. Although there is no clear mechanism behind it, IL- 6 and other inflammatory cytokines may play an important role. Similarly, this phenomenon has also been found in AP, especially obvious in severe acute pancreatitis patients. This is because (a) The reaction in the progress of SAP, including infection, will lead to insulin resistance, which may at last result in metabolic disorders. Thus, the degradation of albumin gets much more for the reason of lower using-rate of glucose and fat; (b) The ability of biosynthesis of albumin in liver is weak for low intake and stimulate of inflammatory factors; (c) In the progress of stress response, vaso permeability become higher so that albumin will permeate into tissue space.
POF is the most important cause of death within the first 2 weeks of disease onset. It's critical to have the ability to assess the risk of AP patients developing. Lots of invasive or non-invasive methods, including biochemical parameters, severity scores and radiological imaging modalities have been applied for predicting POF in patients with AP. In a systemic review for prospective studies, the Bedside Index of Severity in Acute Pancreatitis (BISAP) and the Japanese Severity Score were identified as the best predictors evaluating predictors of POF within $48 \mathrm{~h}$ of admission. However, they were too cumbersome to be applied in the individual patient. This highlights the need to develop approaches to prediction of POF that have early practical utility while still providing a performance sufficient to be applied in the individual patient. Another study found that the Glasgow score is the best classifier at $48 \mathrm{~h}$ of admission for predicting POF in patients with AP by using a head-tohead comparison between the Ranson, APACHE II, Glasgow and the BISAP scoring systems. Mounzer et al. compared several existing clinical scoring systems and found that these scores showed modest accuracy and seemed to have reached their maximal efficacy to predict POF in patients with AP.

In the present research, albumin always descends obviously in AP patients with POF $(p<0.05)$. The AUC under ROC line is 0.869 . Albumin has been proved as an excellent marker of POF in AP. However, no previous study has researched into the association between albumin and incidence of POF in AP. Therefore, this study is the first time to show that the reduction of serum albumin is significantly associated with increased risk of POF in AP.

\section{Conclusion}

Thus it can be concluded that serum albumin on admission is independently associated with POF in AP. The study suggests that albumin is a valuable tool for a rapid assessment of POF in patients with AP. 


\section{Dr. Shuchi Goyal et al, International Journal of Medical and Biomedical Studies (IJMBS)}

\section{References}

1. Hong W, Lin S, Zippi M, Geng W, Stock S, Basharat Z, Cheng B, Pan J and Zhou M. Serum Albumin Is Independently Associated with Persistent Organ Failure in Acute Pancreatitis. Canadian Journal of Gastroenterology and Hepatology. 2017; 10:1155: 10.1

2. Hu X, Zhang L, Advances in antibiotic therapy for acute pancreatitis. Journal Article. 2018; 30:7: 08-711.

3. Hassan AE, Rehim A DESA, Kobiesy, AM, Ashmawy MA, Sayed AAEZ, Ameen SR. Early Predictors of Acute
Pancreatitis Related In-Hospital Mortality: How Practical Are They?Scientific Research. 2018; 8:3: 67-68.

4. $\mathrm{Li}^{1} \mathrm{~S}$, Zhang $\mathrm{Y}, \mathrm{Li}^{2} \mathrm{M}, \mathrm{Xie} \mathrm{C}$ and $\mathrm{Wu} \mathrm{H}$. Serum albumin, a good indicator of persistent organ failure in acute pancreatitis. BMC Gastro. 2017; 17:59: 0615-0618.

5. Li S, Wu H, Zhang Y. Serum Albumin: A Good Indicator of 28-Day-Mortality in Acute Pancreatitis. JACS. Journal of the Am Coll of Surg. 2017; 225:4:2: 128.

6. Mounzer R, Langmead JC, Wu UB, Evans CA, et al. Comparison of Existing Clinical Scoring Systems to Predict Persistent Organ Failure in Patients with Acute Pancreatitis. Gastroenterology. 2012; 142:7: 1476-1482. 